\title{
Bericht über die XI. Versammlung der deutschen otologischen Gesellschaft in Trier am 16. u. 17. Mai 1902. \\ Von
}

Dr. Carl Reinhard in Duisburg am Rhein.

Die diesjährige Versammlung der Deutschen otologischen Gesellschaft, welche damit in ihr zweites Decennium tritt, tagte an den beiden Tagen vor Pfingsten in der gastfreien Moselstadt Trier. Trotz des nichts weniger als maientreundlichen Wetters waren 67 Ohrenärzte aus allen Theilen des deutschen Reiches, Oesterreichs und der Schweiz der Einladung gefolgt und verarbeiteten an den beiden Tagen in 3 Sitzungen (ausser den Vorstandssitzungen) 2 Referate, 14 Vorträge und Demonstrationen.

Der Donnerstag Abend vereinigte bereits eine grosse Anzabl der Collegen theils mit ihren Damen im Gartensaale des Casino, welches auch die Räume zu den wissenschaftlichen Sitzungen und zu den reichhaltigen Ausstellungen chirurgiseher Fachinstrumente bereitwilligst zur Verfügung gestellt hatte.

Anwesend waren die Herren: 1. Auerbach-Baden-Baden, 2. BauerNürnberg, 3. Bezold-München, 4. Bloch-Freiburg i. Br., 5. Brandt, Strassburg i. E., 6. Brieger-Breslau, 7. Burkhoff-Bonn, 8. Delsaux-Brüssel, 9. Denker-Hagen, 10. Dupuis-Trier, 11. Ephraim-Breslau, 12. EschweilerBonn, 13. Farwick-Siegen, 14. Filbry-Coblenz, 15. Hager-Gotha, 16. Hartmann-Berlin, 17. Hartmann-Strassburg, 18. Hecke-Breslau, 19. Hoffmann, R.Dresden, 20. Hübner-Stettin, 21. Ispert-Köln, 22. Jansen-Berlin, 23. JensHannover, 24. Körner-Rostock, 25. Kretschmann-Magdeburg, 26. KurzackCöln (als Gast), 27. Kümmel-Breslau, 28. Lautensehläger-Charlottenburg, 29. Leutert-Giessen, 30. Löhnberg-Hamm, 31. Lindemann-Saarbrücken, 32. Lindt-Bern, 33. Lieders-Wiesbaden, 34. Lüscher-Bern, 35. Mann-Dresden, 36. Meier, Edgar-Magdeburg, 37. Mertens-Münster i. W., 38. Moses-Cöln, 39. Müller-Altenburg (S.-A.), 40. Ostmann-Marburg, 41. Passow-Heidelberg, 42. Poschmann-Trier, 43. Piffl-Prag, 44. Proebsting-Wiesbaden, 45. ReinhardDuisburg, 46. Richter-Magdeburg, 47. Roller-Trier, 48. Roller-Trier, 49. RöllMünchen, 50. Röpke-Solingen, 51. Rudloff-Wiesbaden, 52. Stern-Metz, 53. Scheibe-München, 51. Schlecht-Trier, 55. Schmeden-Oldenburg, 56. SchmitzDuisburg, 57. Siebenmann-Basel, 58. Stacke-Erfurt, 59. Streit-Königsberg, 60. Schwabach-Berlin, 61. Thies-Leipzig, 62. Vohsen-Frankfurt a. M., 63. Wallizek-Breslau, 64. Wiche-Dresden, 65. Wolf, Oscar-Frankfurt a. M., 66. Zarnikow-Hamburg, 67. Zoepffel-Norderney-Wiesbaden. 
Aber so freundlich die Aufnahme auch war, so möchte ich doch gleich an dieser Stelle meinen und vieler anderer Collegen Wunsch ausdrücken, dass die Deutsehe otologische Gesellschaft jetzt aufhört, eine Wanderversammlung zu sein, und nachdem sie, aus den Knabenjahren herausgewachsen, gezeigt hat, was sie kann, welche Stellung sie nunmehr unter den wissenschaftlichen Vereinigungen einnimmt, sich nun auch ein mehr ständiges Heim aussucht, in dem jeder Theilnehmer bereits bei seiner Ankunft zu Hause ist, ohne Rücksicht auf Akustik, Beleuchtung und Temperatur des Sitzungssaales nehmen zu müssen. Es ist für das nächste Jahr Wiesbaden für den Versammlungsort gewählt worden. Wiesbaden ist als alte Congressstadt berühmt, von allen Seiten leicht zu erreichen. Ioh hoffe, dass der XII. Congress uns zu dem Beschluss führt, Wiesbaden als ständigen Versammlungspunkt $z \mathfrak{u}$ bestimmen, wenigstens für alle zwei Jahre; das Zwischenjahr könnte ja dann, wenn absolut noch gereist und geworben werden muss, zum Reisejahr zwisehen Nord und Süd gemacht werden. Die Vortheile der Stabilität des Congressplatzes sind für eine wissenschaftliche Vereinigung hinreichend bekannt, als dass sie nochmals besprochen werden müssten.

Nach Eröffnung der Sitzung am Freitag, den 16. Mai Vormittags $10 \mathrm{Uhr}$ durch den derzeitigen Vorsitzenden Prof. Siebenmann-Basel und nach Begrüssung der Gesellschaft durch den Vertreter der Stadt Herrn Beigeordneten 0 ster und Seitens des Trierer Localcomités begann das Referat von Bezold-München und Körner-Rostock und Piffl-Prag über

\section{Behandlung der acuten Mittelohrentzündung.}

1. Bezold-München.

Bezold folgt gern dem Vorschlage Körner's, welcher mit ihm das Referat über die Behandlung der acuten Mittelohrentzündung übernommen hat, zunächst die ei gene Behandlungsweise zu schildern, will jedoch seine Bedenken auch nicht verschweigen gegen einige gegenwärtig weit verbreitete therapeutische Maassnahmen, die auf Grund langjähriger Beobachtung sich ihm aufgedrängt haben. Der Einfluss der Behandlung tritt am meisten hervor an den genuinen acuten Mittelohrentzündungen, welehe schliessen, während die im Verlauf von schweren Allgemeinerkrankungen, Scharlach, Tuberculose n. s. w. auftretenden Otitiden so viele Abweichungen bieten, dass sie schwer unter einem 
gemeinsamen Gesichtspunkt zu betrachten sind. Die Trommelfellöffnung bei genuiner acuter Mittelohrentzündung bleibt stets eine kleine. Sie liegt meist im hinteren unteren Quadranten, nur ausnahmsweise im vorderen unteren Quadranten. Grössere, mehrfache oder wandständige Perforationen lassen auf eine schwere Allgemeinerkrankung, Tuberculose u. s. w. schliessen.

Die genuine acute Mittelohreiterung endet in der Regel mit Wiederversehluss des Trommelfells und Wiederkehr des normalen Hörvermögens. Typisch in ihrem Verlauf ist nur die ausserordentlich verschiedene Zeitdauer derselben, welche zwischen wenigen Tagen und über Jahresfrist wechseln kann. Die Ursache hierfür sieht Bezold in der ausserordentlich wechselnden Grösse und Ausbreitung der an der Eiterung theilnehmenden Zellen des Schläfenbeins. Sobald diese grossen Höhlen operativ weit eröffnet werden, gestaltet sich auch zeitlich der Heilungsprocess vollkommen typisch und läuft in $3-5$ Wochen ab. Damit ist der Beweis geliefert, dass nur das Höhlenverhältniss in derartigen Fällen die Ursache für die protrahirte Dauer gebildet hat.

Die Aufgaben für die Behandlung der acuten einfachen und perforativen Mittelohrentzündung sind:

1. Die Krankheitsproducte möglichst vollkommen zu entfernen;

2. jede Neuinfection der erkrankten Höhlen hintanzuhalten;

3. dauernd möglichst gnte Abflussbedingungen zu schaffen.

Die erste Aufgabe erfüllt die Luftdouche, für deren uneingeschränkte Anwendung auch bei acuter Entzündung Bezold ebenso eintritt wie seinerzeit v. Tröltsch, und bei stärkerer Secretansammlung die Paracentese des Trommelfells. Das Sichelmesser ist wegen möglicher Verletzung des Bulbus venae jug. zu vermeiden. Auf die Paracentese lässt Bezold regelmässig die Luftdouche, und zwar zunächst vom äusseren Gehörgang, später von der Nase aus folgen.

Um der zweiten Aufgabe zu genügen, macht Bezold täglich einmal eine Injection mit 4 procent. Borlösung, worauf die Luftdouche und gründliche Austrocknung mit Watte umwickelter Sonde unter Spiegelcontrolle folgt. Während des Abtropfens hat der Patient wiederholt den Valsalva'schen Versuch zu machen, um auch die Paukenhöhle möglichst zu entleeren.

Bei grösseren Perforationen im Verlauf von Scharlach, Tuberculose a. s. w. ist ein stärkerer Stempeldruck der Spitze zu vermeiden. 
Wenn Druckempfindlichkeit vorhanden ist, verwendet Bezold den Eisbeutel, kein Kataplasma, auch keine Blutegel. Unter der Borsäurebehandlung wird:

1. das Secret niemals fötid, und bleiben

2. die Gehörwände vollkommen reizlos.

Um der dritten Indication zu genügen, ist die Trommelfellöffnung öfters nach abwärts zu erweitern, Wucherungen auf derselben zu spalten und mit der Schlinge abzutragen oder eine neue Oeffnung im Trommelfell anzulegen. Aetzungen und Galvanokaustik vermeidet Bezold am Trommelfell.

Wenn die acute Mittelohrentzündung trotz correcter Behandlung länger als zwei Monate andauert, so hält Bezold die Annahme einer Complication für gesichert und macht die Eröffnung des Warzentheils, auch zögert er nicht mit der Eröffnung bei Schwellung der äusseren Weichtheile, insbesondere unterhalb des Warzenfortsatzes. Im letzteren Falle liegt die Durchbruchsstelle des Eiters am Boden des Warzenfortsatzes manchmal so tief, dass man bis zum Bulbus ven. jug. vorgehen muss. Ausspülungen der Mittelohrräume durch den in die Tuba eingeführten Katheter verwirft Bezold bedingungslos; auch ïber die schädlichen Wirkungen der gegenwärtig vielfach geübten trockenen Gehörgangstamponade theilt Bezold eine Reihe von Beobachtungen mit.

\section{Körner-Rostock.}

Der Correferent Körner-Rostock erwähnt die Einwände, die von $\mathrm{Z}$ a u fal und dessen Schüler Piffl gegen die Paracentese des Trommelfells erhoben worden sind, und überlässt es dem anwesenden Herrn Piffl, dieselben der Versammlung darzulegen. Er ist ein entschiedener Gegner dieser Zaufal-Pifflschen Lehre, und zwar aus theoretischen Gründen ebenso sehr wie auf Grund seiner klinisehen Erfahrungen. Die Entscheidung in solchen therapeutischen Fragen wird aber nach seiner Ueberzeugung weniger durch unsere wechselnden theoretischen Anschauungen, sondern vorzugsweise durch die klinische Erfahrung gefällt. Diese spricht heute noch ebenso wie vor dem Auftreten von $Z$ a u fal und $P$ iffl auf das Dentlichste zu Gunsten der frïhzeitigen Paracentese. Der Redner schildert zunächst die auf seiner Klinik übliche Behandlungsweise der acuten Mittelohrentzündung, die sich nicht an ein starres Schema hält, aber im Wesentlichen nach folgenden Principien gehandhabt wird. Die Vornahme der Paracentese wird nöthig, sobald ein entzündliches 
Exsudat in der Paukenhöhle erkennbar ist; wenn erst die Symptomentrias: Vorwölbung des Trommelfells, Fieber und Sehmerzen vorhanden ist, giebt es keine Entschuldigung für Unterlassung der Paracentese. Nach derselben wird der Gehörgang mit einem Gazestreifen drainirt und ein aufsaugender Verband angelegt. Der Versuch, den Gehörgang vor der Paracentese zu desinficiren, ist ganz vergeblich und unterbleibt deshalb. Können wir doch nicht einmal unsere der Desinfection leicht zugänglichen Hände absolut keimfrei machen. Gegen die Secundärinfection vom Gehörgange aus schützt der aseptische Verband, wie die Erfahrung lehrt, mit fast absoluter Sicherheit. Bettruhe ist erforderlich. Auch die kranke Paukenhöhle muss in Ruhe gelassen werden, namentlich dürfen keine Lufteintreibungen von der Tube aus vorgenommen werden. Es ist dafür Sorge zu tragen, dass der Abfluss des Eiters durch die angelegte Trommelfellöffnung durch nichts behindert wird. Aseptische Ausspülungen des Gehörgangs sind nur bei sehr zähem, schleimig-eitrigem Secrete nothwendig. Therapeutische Maassnahmen aussen am Warzenfortsatze (Blutegel, Jodanstrich) bringen keinen Nutzen, sondern machen Hautentzündungen, die eine etwa complicirende Mastoiditis verbergen. Bei starker Sehmerzhaftigkeit und Periostschwellung am Warzenfortsatze wird Eis applicirt, jedoch nur in sehr schweren Fällen, da die Anwendung des Eises den Verband unmöglich macht, der schon aus Reinlichkeitsgründen nothwendig ist.

Von 78 auf solche Weise behandelten Fällen ist nur einer, der durch eine Gehörgangsstenose complicirt war, chronisch geworden. Die anderen sind alle mit Versehluss der Trommelfellöffnung und völliger Erhaltung des Gehörs geheilt. Es zeigt sich ferner, dass die Heilung um so schneller erfolgte, je früher die Paracentese gemacht worden war. Im Durchschnitt war die Heilung vollendet

$$
\begin{aligned}
& \text { am 7. Tage bei Paracentese am 1. Tage }
\end{aligned}
$$

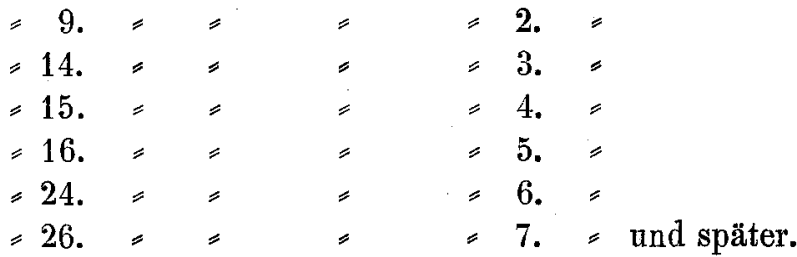

Viel später erfolgte die Heilung in 42 Fällen aus der gleichen Beabachtungszeit, bei welchen die primäre Paracentese nicht 
vorgenommen werden konnte, weil die Kranken erst nach erfolgtem Spontandurchbruch in die Behandlung eingetreten waren. Auch hierbei zeigte es sich, dass die frühe Entlastung der Paukenhöhle durch frühzeitigen Spontandurchbruch schneller zur Heilung führte, als durch spät erfolgtem, und zwar durchschnittlich am 14. Tage bei Spontandurchbruch am 1. Tage

$=17 .=$
$=21 .=$
$=26 .=$

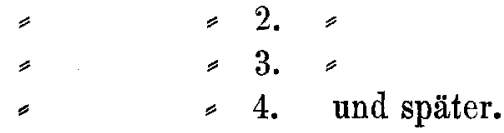

Dass die frühzeitige Paracentese den besten Schutz gegen eine complicirende Mastoiditis gewährt, zeigt folgende Erfahrung. Von 90 in gleichem Zeitranme operirten Mastoiditiden waren nur 19 paracentesirt worden, und von diesen nur 4 rechtzeitig, d. h. am 1. oder 2. Tage. Und in einer ununterbrochenen Reihe von 405 operirten acuten und ehronischen Mastoiditiden waren nur 43, bei denen die Paracentese gemacht worden war. In dem gleichen Zeitraume sind in der Klinik mehr als 1200 Paracentesen gemacht worden. Wäre die Paracentese ein nachtheiliger Eingriff, so müssten viel mehr vorher paracentesirte Mastoiditiden beobachtet worden sein, da in Rostock und in ganz Mecklenburg keine Concurrenzklinik besteht, an die sich etwa die ungünstigen Fälle gewendet haben könnten.

\section{Piffl-Prag. Zur Behandlung der Otitis media a cuta.}

Der Vortragende beschreibt die historische Entwicklung, die Anwendungsweise und die Erfolge der von $\mathrm{Zau}$ fal angegebenen seit mehr als einem Decennium an seiner Klinik im Gebrauch stehenden Behandlungsmethode acuter Mittelohrentzündungen. Gegründet auf die Erkenntniss, dass diese Erkrankung einen cyklischen Verlauf besitzt, welcher um so günstiger abläuft, wenn eine Perforation des Trommelfells, sei sie spontan oder künstlich, nicht eintritt, strebt die Behandlung Linderung des Schmerzes, Verminderung der Ausscheidung und Unterstützung der Resorption des Paukenhöhlensecretes an, und zwar durch heisse Einpackungen mit essigsaurer Thonerde, innerliche Anwendung von Salicylpräparaten, Einpinselungen mit Jodtinctur, Einträufelungen von Cocaïnlösungen und mitunter Massage in der Umgebung des Ohres. Unter dieser Behandlung lassen die Schmerzen und die Entzündungserscheinungen nach, und eine Paracentese wird nur selten nothwendig. Die Entstehung von Complicationen 
ist bedingt durch hochgradige Virulenz oder besondere Eigenthümlichkeiten des Erregers oder durch ungünstige allgmeine oder locale anatomische oder pathologisch-anatomische Verhältnisse. Der Vortragende erinnert hier an die für den Abfluss von Secret aus dem Warzenfortsatze absolut ungeeignete Anordnung und die unguinstigen Volumverhältnisse der pneumatischen Zellen. Entsprechend den Verhältnissen, die eine Entzündung im Warzenfortsatze antrifft, kann man zwei Gruppen von Entzuindungen unterscheiden. Die erste Gruppe, die Mehrzahl der genuinen Entzündungen, insbesondere die dureh Hyperplasie der Rachenmandel bedingten, verlaufen unter der angegebenen $\mathrm{Be}$ handlung guinstig, ohne dass eine Paracentese nothwendig würde. Sie scheinen sogar, wie zahlreiche Beobachtungen ergeben haben, durch die Perforation ungünstig beeinflusst zu werden, abgesehen davon, dass dadureh die Möglichkeit der secundären Infection und der Cholesteatombildung (Bezold) gegeben ist. - Die zweite Gruppe bilden Fälle, die von Anfang an schwer verlaufen, auch trotz frïhzeitiger Paracentese. Dieser Eingriff ist in diesen Fällen nutzlos, weil der Hauptherd der Erkrankung nicht mehr in der Paukenhöhle, sondern in den Zellen des Warzenfortsatzes liegt. Es wird aber, wenn trotz entsprechender Behandlung am 7. oder 8. Tage kein Riickgang der Erscheinungen eintritt, ein Versuch mit der Paracentese gemacht, Sind jedoch bereits deutliche Erseheinungen einer Complication vorhanden, dann wird, ohne mit der Paracentese unnutze Zeit zu verlieren, die Aufmeisselung des Warzenfortsatzes vorgenommen. Trotz der in Folge dieser strengen Indicationsstellung geringen Anzahl von Paracentesen ist an der Zaufal'sehen und anderen, z. B. der Siebenmann'schen Klinik, keine Vermehrung der Aufmeisselung zu beobachten.

Discussion: Als Erster erhält das Wort unser Gast: Herr Delsaux-Brüssel berichtet ïber die Erfahrungen der belgischen Ohrenärzte und über die in einer Versammlung der Soeiété Française de Laryngologie ete. in Paris kurz zuvor über denselben Gegenstand stattgehabten Verhandlungen. Im Allgemeinen sprach sich die Versammlung für die frühzeitige Paracentese aus, ohne Lufteinblasung und ohne Einspritzungen. Nach 2-3 Wochen bei profuser Eiterung bei Mastoiderscheinungen soll die Warzenfortsatzoperation vorgenommen werden. Es wurde beschlossen, eine Commission einzusetzen, welche in zwei Jahren berichten soll. 
Vohsen-Frankfurt a. M. betont die Lagerung der Kranken bei acuter Mittelohreiterung wie folgt:

Die Referate haben, wie auch die Lehrbücher, eine Frage unberührt gelassen, die mir bei Behandlung der Mittelohrentzündungen von besonderer Wichtigkeit erscheint und über die wir uns verständigen sollten: Wie sollen wir den Kranken mit Mittelohrentzündungen lagern? - Meist wird dem Kranken angerathen, sich auf die kranke Seite zu legen. Gerade das Gegentheil davon ist richtig. Die Entlastung des kranken Ohres und die wichtige Drainage, auf die alle mit Recht einen grossen Werth legen, wird am besten erreicht durch Lagerung des Kranken auf die gesunde Seite.

Bei einseitiger acuter Mittelohrentzündung mit und ohne Perforation erreichen wir durch Lagerung auf die gesunde Seite erstens eine Verminderung der Blutfulle auf der kranken Seite und eine entsprechende subjective Erleichterung, zweitens begünstigen wir das Wegsamwerden der Tube durch Abschwellung und sorgen so für Ventilation des Mittelohrs, vorzugsweise den Abfluss von Secreten auf diesem Weg, drittens schïtzen wir so am besten das Antrum und die peripheren Warzenzellen, in die bei entgegengesetzter Lage das Secret geradezu hineingetrieben wird. - Auch der Kuppelraum entleert sich am leichtesten bei der empfohlenen Lage. -

Bei mancher Mittelohrentzündung habe ich dem Kranken, der ängstlich die von anderer Seite empfohlene Lage anf dem kranken Ohre einhielt, allein durch Anordnung der richtigen Lage grosse Erleichterung geschafft.

Bei doppelseitiger Erkrankung empfiehlt sich die Lagerung auf die weniger erkrankte Seite oder ein Wechsel in der Seitenlage. Die Rückenlage begünstigt aus naheliegenden Gründen das Fortschreiten der Erkrankung nach dem Antrum und dem Sinus.

Leutert-Giessen vertritt den Schwartze'schen Standpunkt, frühzeitige breite Paracentese betreffend und Ruhe des Kranken, wendet sich aber 1. gegen die Pulverbehandlung; 2. gegen das regelmässige Ausspritzen; 3. gegen das regelmässige Austupfen.

1. Die Pulverbehandlung hat keinen Zweck, da sie nur das Trommelfell, nicht aber den eigentlichen Krankheitsherd, die Paukenschleimhaut trifft. Wenn Schwartze diese Behandlung verwirft, so hat er dazu seine guten Gründe gehabt. Er hat 
nicht selten Fälle gesehen, wo die Pulverbehandlung bei ge$r$ inger Eiterung angewandt zur Verklebung der Perforation geführt hat. Wurde dann die Eiternng stärker, so gab diese ein Hinderniss für den Eiterabfluss ab. Leutert ist ausserdem gegen die Pulverbehandlung, weil sie für den praktischen Arzt ein zu bequemes Mittel ist. Dieser bemüht sich nicht mehr, die Trommelfellperforation, resp. Eiterverhaltung zu erkennen, da ja "doch nur Pulver eingeblasen wird"; dass das Ohr eitert, sieht er ja, also bläst er immer frisch Pulver ein. Er hat Fälle gesehen (mit oder ohne Eiterung), in denen beide Gehörgänge ganz mit Borpulver angefüllt waren. Dieses giebt natürlich ein Hinderniss für den Eiterabfluss ab. Das regelmässige Spritzen hat auch keinen Zweck, denn es entfernt nur den Eiter, der den Krankheitsherd bereits verlassen hat, desgleichen das sorgfältige 'Tupfen, welch letzteres ausserdem reizt.

Kretschmann-Magdeburg: Es ist mit Freuden zu begrüssen, dass in den Referaten der frühere Dualismus von acutem einfachen Katarrh und acutem eitrigen Katarrh, der unnatürlich war, beseitigt ist. Bei der Anwendung der Paracentese ist zu berücksichtigen die Thatsache, dass die meist grössere Anzahl der acuten Otitiden ohne Durchbruch heilt. Der Befund eines vorgewölbten, gerötheten Trommelfelles, die Anwesenheit von leichten Fiebersteigerungen berechtigt noeh nicht ohne Weiteres zur Paracentese. Bei zweckmässigem Verbalten gehen diese Symptome oft überraschend schnell zurtick. Die Vorwölbung ist keineswegs immer ein Zeichen für Secretansammlung; die geschwollene Schleimhaut als solche drängt das Trommelfell vor. Bei manchen Paracentesen sieht man gar kein Secret aus dem Schnitt austreten, nur Sehleimhaut prolabiren. Es bleibt zu erwägen, ob nicht der Druck der geschwollenen Schleimhautflächen, wenn sonst die Entzündung ihren Höhepunkt überschritten hat, günstig für die Rückbildung wirkt. Die Paracentese hebt aber den Druck auf. Eine Paracentese auf den ersten Blick ist jedenfalls bei frischer Entzïndung nur in den seltensten Fällen gerechtfertigt. Abwarten von 24 Stunden, eventuell länger, lässt den Fall klarer beurtheilen.

Ist Durchbruch eingetreten, so ist die Einblasung von Borsäure zu widerrathen. Die Thatsache, dass in ungeübter Hand dadurch Schaden gestiftet wird, ist des Oefteren zu verzeichnen. Um die antizymotische Wirkung des Borpulvers ohne Schaden 
zu benutzen, kann man Gazebäuschchen in Borpulver wälzen und damit in den Gehörgang führen.

Kretschman n-Magdeburg führt einen Todesfall von Meningitis ex otitide acuta an, bei welchem der Patient gegen seineAnordnung Borpulver in grossen Mengen in den Gehörgang eingeblasen und dadurch Eiterretention mit tödtliehem Ausgang hervorgerufen hatte.

Ostmann-Marburg vertritt denselben Standpunkt; frühzeitige Paracentese, Occlusivverband, keine Insufflation, aber auch keine Katheterluftdouche.

Passow-Heidelberg macht die Paracentese so gross als möglich im hinteren Trommelfellabschnitt.

0 . Wolff-Frankfurt a. M. hat von der Alkoholbehandlung gute Erfolge gesehen.

Lautenschläger-Charlottenburg macht auf den Bakteriengehalt der äusseren Luft aufmerksam, welche durch die Luftdouche in das Ohr getrieben werden, und empfiehlt nach seiner Methode, die Luft za sterilisiren und dann einzublasen.

Scheibe-München vertritt den Bezold'schen Standpunkt.

Jens-Hannover legt im hinteren oberen Quadranten einen Kreuzschnitt an; ein derartiger Schnitt klafft besser als jeder andere und verklebt auch nicht so schnell wieder, wie ein einfacher linearer Schnitt; ungünstige Narbenbildung, resp. ungünstiger Einfluss auf die spätere Hörfähigkeit hat er dabei nicht beobachtet. Mit der frühzeitigen Discision kann er sich jedoch nicht befreunden; er sieht die meisten Fälle bei conservativer Behandlung - Einträufelung von Carbolglycerin-Abführmittel, eventuell Blutegel, Ruhe - zurückgehen; nicht selten kommt es dabei zu Mastoiditis. Den Katheter wendet Jens sehr selten an, erst wenn das Trommelfell abgeblasst ist. Zur Entfernung des Secretes durch die Trommelfellöffnung hindurch bedient er sich des Siegle'schen Trichters und der Delstancheschen Luftpumpe, die ihm gute Dienste gethan haben.

Brieger-Breslau: Für die Therapie der acuten Mittelohrentzündung muss der Gesichtspunkt leitend bleiben, dass es sich um eine gewöhnlich zu spontanem Ablauf tendirende Krankheit handelt. Es sind daher in erster Linie alle Behandlungsmethoden darauf zu prüfen, ob sie geeignet sind, diesen Spontanablauf irgendwie ungünstig zu beeinflussen. Von den Methoden der Abortivbehandlung leistet allein die Application von Blutegeln etwas. Wird dadurch die Entwickelung der Otitis media nicht aufgehalten, so wird doch anscheinend wenigstens der Verlauf 
günstig beeinflusst. Der Werth der Kälteapplication wird überschätzt. Ihr symptomatiseher Werth für manche Fälle ist unbestritten. Auf den Ablauf des entzündlichen Processes bleibt sie schon deswegen ohne wesentlichen Einfluss, weil sie, auf die Umgebung des Ohrs applicirt, in den Mittelohrräumen kaum noch wirksam ist. Temperaturmessungen in der Pauke bei radical operirten Patienten, bei denen die Operationswunde geschlossen war, ergaben, dass durch äussere Eisapplication die Temperatur in der Pauke kaum herabgesetzt wurde. Einer anfänglichen Steigerung der Temperatur folgte eine meist minimale Erniedrigung, welche bei Weglassen des Eisbeutels sofort wieder verschwand. Eisanwendungen für Stunden ist also in jedem Falle nutzlos. Auch durch Wärmeapplication wird die Temperatur nicht mehr als um einige Decigrade in die Höhe getrieben. Der Entzündungsprocess würde also - ganz abgesehen davon, dass nenere Versuche einen besonderen Einfluss der Wärme auf den Ablauf desselben zu erweisen scheinen - nicht wesentlich beeinflusst werden, wenn man zur Linderung von Schmerzen Wärme äusserlich anwendete.

Bei allem Respect vor der Autorität Zaufal's möchte Brieger die Gesichtspunkte nicht verschieben lassen, die seit der Einführung der Paracentese durch Schwartze maassgebend sind. Die durch die Paracentese bergestellte Communication der Panke mit dem Gehörgang reicht an sich nicht aus, eine Umwandlung seröser Transsudate in eine Eiterung herbeizuführen. Eine Desinfection des Gehörgangs ist, wie frühere Versuche Brieger's gelehrt haben, undurchführbar, ohne erhebliche Beschwerden auszulösen, und entbehrlich. Bei Entzündungen mit zapfenförmiger Perforation ist immer an eine besondere Form oder Localisation der Entzündung zu denken; daher werden diese Perforationen durch Incisionen im hinteren unteren Quadranten nicht beeinflusst. - Bedenklich sind in der Nachbehandlung nach der Paracentese nur diejenigen Methoden, bei denen der Transport der Gehörgangsepiphysen, von deren Einwanderung die Chronieität der Eiterung nach Le utert wesentlich abzuhängen scheint, in die Pauke befördert wird. Daher sind Spülungen wesentlich gefährlicher, als die Anwendung des $\mathrm{Ka}-$ theters. Versuche mit im Nasenrachenraum verstäubten Farbstoffpartikeln ergaben, dass diese durch den Katheterismus nicht in die Panke befördert werden. Nicht auszuschliessen ist dagegen die Verbreitung von Keimen innerhalb der einzelnen $\mathrm{Ab}$ - 
schnitte des Mittelohrs. Die Durchspülung per tubam ist unbedenklich, wenn man sich strict an die von Schwartze aufgestellten Indicationen hält und sie somit für die Dauer des Bestehens frisch entzündlicher Erseheinungen ausschliesst.

Gegenüber der Bemerkung, dass zur Antisepsis kräftige Injeetionen nöthig seien, bemerkt Brieger, dass dadurch nur Erreger in die Pauke hinein, nicht aber aus der Pauke und Gehörgang heraus befördert würden. Der Werth der Gehörgangstamponade scheint überschätzt zu werden. Sie leistet für die Drainage der Pauke nichts, schützt im Wesentlichen nur durch Aufsaugung des Secretes gegen Dermatitiden der Gehörgangswände.

Die Festsetzung bestimmter Maximalfristen, innerhalb deren die Secretion erloschon sein müsste, erscheint bedenklich. Damit, dass bei Aufmeisselungen in den ersten Wochen erheblich grössere und schwere Veränderungen anfgedeckt werden, als man vermuthet hatte, wird nicht bewiesen, dass diese Veränderungen nicht hätten gleichwohl rückgängig werden können. Die Indication für die Eröffnung der Paukennebenräume ist lediglich von der Anwesenheit der für die Betheiligung dieser Nebenräume charakteristischen Befunde, nicht aber von der Dauer der Eiterung abhängig.

Kümmel-Breslau: Meiner Meinung nach kann eine statistische Bearbeitung der Resultate bei bestimmter Behandlungsweise der acuten Otitis nur dann einen Werth haben, wenn man streng unterscheidet zwischen den Entzündungen, die sich im "freien Paukenhöhlenraum" (dem den beiden vorderen and dem hinteren unteren Trommelfellquadranten entsprechenden Theil der Paukenhöhle) abspielen, und denen, die in dem Abschnitt localisirt sind, der dem hinteren oberen Quadranten entspricht. Letztere Form ist dadurch von viel grösserer klinischer Bedeutung, dass hier die Wände der Höhle eine ganze Anzahl Nischen besitzen, und der Hohlraum ausserdem von zahlreichen Bandsträngen durchzogen ist, die die Entzündungserreger und Entztindungsproducte gewissermaassen fixiren, so dass viel leichter eine schwerere Erkrankung der Nebenräume der Paukenhöhle entstehen kann. Die im freien Paukenhöhlenraum localisirten Entzündungen sind für den Organismus wie für die Hörfunction viel weniger bedeutungsvoll, heilen auch viel leichter, mit oder ohne Perforation. Die andere Form bedarf von vornherein der ernstesten Beobachtung. Heilen können beide Formen mit oder ohne Perforation, je nach der absoluten oder relativen (im Ver- 
hältniss zur Widerstandsfähigkeit des Gesammtorganismus und der Paukenhöhlenschleimhaut) Virulenz der betreffenden Entzündungserreger. Complicationen treten aber nur bei der „epitympanalen Form" häufiger auf und erlangen nur bei ihr eine schwerere Bedeutung. Wohl bei jeder acuten Otitis ist das Epitympanum von vornherein betheiligt, die Entzündung daselbst wird aber ausheilen, wenn die Entleerung der dort gebildeten Entzündungsproducte nicht durch ungünstige anatomische Verhältnisse verhindert wird. Solche liegen aber vor, wenn das erwähnte Balkenwerk stark ausgebildet und durch stärkere entzündliche Sehwellung die Mächtigkeit der betreffenden Schleimhautfalten noch vermehrt ist. Dann sperren diese Balken das Epitympanum ab. Entspannt man in solchen Fällen aber durch eine frühzeitige, sehr ausgiebige Paracentese diese schwammige Partie, oder schaffen die Entzündungsproducte sich frühzeitig durch eine ausgiebige Spontanperforation einen Ausweg; so tritt diese Störung zurïck. Die beutelförmige Gestalt der Vorwölbungen, auf deren Kuppe die Spontanperforation einzutreten pflegt, kann aber auch dann noch einen ventilartigen Abschluss dieser Balkenräume bewirken, der manchmal verschuldet, dass die Entzündungsproducte trotz der Perforation dauernd unter erhöhtem Druck stehen. (Analogie zur Urinretention bei Ischuria paradoxa.)

Ob die eine oder die andere Form der Otitis auftritt, hängt sicher zum Theil ab von anatomischen Eigenthümlichkeiten der erkrankten Paukenhöhle, zum Theil von mechanischen Zufälligkeiten beim Eindringen der Entzündungserreger, ausserdem wohl noch von manchen uns zum Theil noch ganz unbekannten Factoren. Jedenfalls erscheint es mir aber nothwendig, bei der Pathologie und Therapie der acuten Otitis nicht nur auf die ursächlichen Mikroorganismen, sondern auch auf den erkrankten Organismus die Aufmerksamkeit zu lenken. Manche Differenzen zwischen den Anschauungen verschiedener hochgeschätzter Obrenärzte werden verschwinden, sobald man die eitrige Otitis media nicht als ein einheitliches Krankheitsbild, sondern als eine Summe von Erkrankungen sehr verschiedener klinischer Bedeutung auffasst, die gesondert betrachtet werden müssen. Eigentlich erscheint es mir danach am besten, wenn wir dem Beispiel der französisehen Gesellschaft folgen und dasselbe Thema noch einmal in 2 Jahren zur Discussion bringen würden, nachdem vorher von einer Commission die in dieser Diseussion zu Tage ge- 
tretenen Gesichtspunkte und Meinungsdifferenzen noch einmal studirt worden sind.

Jansen-Berlin: Leider kann ich nur auf den Vortrag des letzten Redners (Piffl) eingehen, da ich bei den Vorträgen der Herren Referenten Bezold und Körner noch nicht zugegen war. Die Paracentese halte ich für sehr segensreich und will sie lieber ein Mal zu viel machen, als sie in einem Falle unterlassen, in dem sie nöthig ist. Wenn man die Paracentese unter allen Cautelen ausführt, so schadet man niemals damit, nützt aber vielfach ausserordentlich. Wir behandeln nicht die Krankheit, sondern den Kranken. Wo schwere Symptome sind, miissen wir anders handeln als bei leichten Erkrankungen ohne Beschwerden. Es liegt ein Körnchen Wahrheit darin, dass die Fälle ohne Durchbruch leichter verlaufen; denn die leichten Formen der Entzündung führen eben gar nicht zur Eiterung und zum Durchbruch. Mein Standpunkt in dieser Frage ist genau derselbe, wie ieh ihn in meiner Arbeit über Otitis media in der Deutschen Klinik vor einigen Monaten eingenommen habe. Die ausgedebnte Paracentese, wie sie Passow macht, durch den ganzen hinteren Trommelfellabschnitt von oben nach unten halte ich nicht nur für unnöthig, sondern auch für bedenklich aus Rïcksicht auf den Amboss.

Si ebenmann-Basel will vor Allem individualisirt haben und empfiehlt die Paracentese in erster Linie bei Kindern, bei denen sie grossen Nutzen bringt, sodann Bettruhe und Eisbeutel. Er sowohl als Esehweiler-Bonn, welcher aus Erfahrung am eigenen Leibe spricht, betonen die Wichtigkeit, dass die Anwendung der Kälte permanent sein müsse und nicht unterbrochen werden dürfe. Am Tage soll eine Eisblase die andere ablösen ohne Unterbrechung bis spät Abends. Nachts findet dann der Kranke sowohl als die Umgebung die nöthige Ruhe.

Bezold hat bei Lufteinblasungen von der Tuba aus keine Infection gesehen; die Paracentese, wenn nöthig, bietet keine Gefahr, ebensowenig die Aspiration des Secretes von aussen.

Körner: Die Symptomentrias: Fieber-Schmerz-Vorwölbung indicirt sofortige Paracentese, und diese bietet dann die besten Chancen für eine Restitutio ad integrum.

Piffl billigt diesen Standpunkt nicht; es wird zu viel paracentesirt. Durch warme Umschläge und innerliche Verabreichung: von salicylsaurem Natron wird dasselbe erreicht. Die günstigen Resultate, die Körner durch Paracentese am 1. oder 2. Tage 
erzielt hat, können darauf zurückzufuhhren sein, dass es sich zufällig um leichte Fälle gehandelt hat, die auch ohne Paracentese zum Mindesten ebenso rasch geheilt wären. Gegenüber den Bemerkungen Leutert's und Jansen's muss daran festgehalten werden, dass die $\mathrm{Z}$ a $\mathrm{u}$ fal'sche Behandlungsmethode nicht nach der geringen Anzahl der Paracentesen beurtheilt werden darf. Es ist ein Effect unserer Behandlung, dass die he ute von $\mathrm{Kör}$ ner als Indication zur Paracentese aufgestellte Trias: Schmerz, Fieber und Vorwölbung des Trommelfells, oft binnen Kurzem nicht mehr vorhanden ist, dass daher die Paracentese entbehrlich wird. Dass dabei nicht schematisirt werden darf, vielmehr jeder Fall nach seinem Verlauf beurtheilt werden muss, ist für den denkenden Arzt wohl zweifellos.

In der Mittagspause folgte die Versammlung einer Einladung der Stadt Trier zu einem Frühstück in den unteren Räumen des Casino, bei welchem Osear Wolff-Frankfurt a. M. Gelegenheit nahm, um mit warmen Worten den Dank der Gesellsehaft für den überaus gastlichen Empfang, den die Stadt Trier ihr grewähre, auszusprechen.

II. Die Unfallverletzungen des Gehörorganes und die procentuale Abschätzung der durch sie herbeigeführten Einbusse an Erwerbsfähigkeit im Sinne des Unfallversicherungsgesetzes.

Röpke-Solingen (Referat).

I. Häufigkeit der Unfallverletzungen des Gehörorgans.

Unter 45971 Betriebsunfällen der gewerblichen Berufsgenossenschaften, die im Jahre 1897 entschädigt wurden, waren 57 Fälle $=1,24$ pro mille, in denen Folgen von Verletzungen des Gehörorganes die Rente bedingten. Den grössten Procentsatz (25 unter $5670=4,41$ pro mille) batte die KnappschaftsBerufsgenossensehaft. (Acten des Reichs-Versicherungsamtes.)

II. Entstehungsart der Ohr-Unfallverletzungen.

Die Entstehungsart von Unfallverletzungen der Ohrmuschel, des äusseren Gehörganges, des Trommelfelles, des Mittelohres und des inneren ?Ohres werden nacheinander besprochen; dabei werden selbst beobachtete oder in Recursentscheidungen des Reichs-Versicherungsamtes enthaltene Grenzfälle eingefügt.

III. Welche Folgezustände nach Unfallverletzungen des Gehörorganes können eine zeitweise oder 
dauernde völlige oder theilweise Erwerbsunfähigkeit des Verletzten im Sinnedes Unfallversicherungsgesetzes herbeiführen?

a) Durch Unfallverletzungen entstandene Verkrippelungen der 0 hrmuschel bedingen in der Regel keine Rente. Dagegen ist die Entstellung bei Verlust einer Ohrmuschel so gross, dass eine geringe Rente am Platze ist.

b) Nach Verletzungen des äusseren Gehörganges können so hoehgradige narbige Veränderungen des Gehörorganes zurückbleiben, dass Schwerhörigkeit und subjective Geräusche dadureh bedingt werden.

c) Trommelfell- und Mittelohrverletzungen, die ohne Eiterung heilen, hinterlassen nur zum Theil so hochgradige Schwerhörigkeit, dass durch diese Unfallsfolge eine theilweise Erwerbsunfähigkeit herbeigeführt wird. Ist eine Eiterung eingetreten, so kann eine Dauerrente erst dann festgesetzt werden, wenn die Eiterung beseitigt ist, oder, wenn diese nach dem Gutachten des Arztes nicht beseitigt werden kann. (Operative Behandlung kann nur mit Zustimmung des Verletzten eingeleitet werden.) Bei chronischer Eiterung können Sehwerhörigkeit, Schwindelerscheinungen, Kopfschmerzen, subjective Geräusche u. s. w. die Erwerbsfähigkeit der Verletzten erheblich herabsetzen.

d) Als Unfallsfolgen von Verletzungen des inneren 0 hres bleiben meistens hochgradige Schwerhörigkeit oder Taubheit, Schwindelerscheinungen, Kopfschmerzen und subjective Geräusche zurück. Die Beurtheilung solcher Fälle ist nur dem Arzt möglich, der mit sämmtlichen Untersuchungsmethoden, die zur Erkennung von Erkrankungen des inneren Ohres Anwendung finden, vollständig vertraut ist. Es ist zu empfehlen, dass Unfallversicherte, die Verletzungen des Schädels erlitten haben, möglichst bald nach dem Unfall von einem Ohrenarzt untersucht werden.

IV. Die procentuale Absehätung der dureh Unfallverletzungen des Gehörorganes herbeigeführten Einbusse an Erwerbsfähigkeit.

Wir haben streng zu individualisiren: der Beruf, das Gesehlecht, das Alter, der allgemeine körperliche und geistige Gesundheitszustand des Rentenanwärters sind Factoren, die bei der Festsetzung der Rentenhöhe mit in die Wagschale fallen. Als entschädigungspflichtige Folgezustände nach Unfallverletzungen des Gehörorganes kommen hauptsächlich in Betracht: 
Bericht über die Versammlung der deutschen otologischen Gesellschaft. 99

1. Herabsetzung oder Verlust des Hörvermögens mit oder ohne Ohreiterung;

2. Schwindelerscheinungen, objective Geräusche, Kopfschmerzen;

3. Entstellungen.

ad 1. Es ist zu unterscheiden zwischen einseitiger und beiderseitiger Schwerhörigkeit oder Taubheit. Eine Beschränkung. der abstracten Erwerbsfähigkeit tritt bei dem Durchschnittsarbeiter ein, wenn er nicht mehr im Stande ist bei vollständig normalem Hörvermögen auf der einen Seite Fltistersprache, auf dem anderen Ohre auf 4 Meter Entfernung zu hören. Bei einseitiger hochgradiger Schwerhörigkeit ist eine Zubilligung von 10 Proc. der Vollrente am Platze. Besteht einseitige Taubheit, so ist auf mindestens 20 Proc. zu erkennen. Ist die angegebene Mindesthörschärfe beiderseits nicht vorhanden, so ist es praktiseh, den Procentsatz der Erwerbsunfähigkeit unter Berücksichtigung des speciellen Falles für jedes Obr einzeln abzuschätzen; durch Addition dieser beiden Werthe wird die in Wirklichkeit eingetretene Schädigung annähernd richtig getroffen werden.

ad 2. Bei der Abschätzung von Schwindelerscheinungen kommt es darauf an, ob der Verletzte noch im Stande ist, auf Leitern zu klettern, auf Gerüsten zu arbeiten, ob er noch im Stehen arbeiten kann, ob er sich bücken kann, wie lange er arbeiten kann und in weleher Güte er die ihm übertragenen Arbeiten noch ausführen kann. Die durch Schwindel herbeigeführte Einbusse an Erwerbsfähigkeit ist auf mindestens 10 Proc. abzuschätzen. Sind heftige objective Geräusche zurückgeblieben, so dass sie die Nachtruhe des Verletzten beeinträehtigen, oder werden diese Geräusche bei länger anhaltender Arbeit oder im Geräusch des Fabrikbetriebes unerträglich, so ist die durch diese Unfallfolge herbeigefuhhrte Einbusse an Erwerbsfähigkeit ebenfalls auf mindestens 10 Proc. abzuschätzen.

ad 3. Für Einstellung, die dureh Verlnst der Ohrmusehel eintritt, ist mindestens 10 Proc. der Vollrente zuzubilligen.

Bestehen verschiedene der oben besprochenen Unfallfolgen neben einander, so würden diese nach dem oben angegebenen Princip einzeln in ihren Einwirkungen auf die Erwerbsfähigkeit des betreffenden Individuums abzuschätzen und der Werth zu addiren sein. 
Discussion:

Reinhard-Duisburg betont die Wichtigkeit, die Ohrenunfallverletzungen möglichst frühzeitig in Beobachtung und Behandlung zu bekommen, um in der Lage zu sein, uberhaupt feststellen zu können, ob und in wie weit der Unfall Folgen für das Ohr des Betreffenden gehabt hat. Er reicht eine Resolution herum, welche die westdeutsche Vereinigung der Hals- und Ohrenärzte in Cöln auf seine Veranlassung hin gefasst und an alle 103 Berufsgenossenschaften des deutschen Reiches gesandt hat. Die Resolution lautet:

"Es ist von den Mitgliedern unseres Vereins wiederholt beobachtet worden, dass Ohrenunfallverletzte fast stets erst so spät nach dem Unfall dem Ohrenarzt behufs Begutachtung zugeführt werden, dass es dem Ohrenarzt schwer, in vielen Fällen sogar unmöglich ist, eine sichere Entscheidung zu treffen oder den Grad der Erwerbsbeeinträchtigung in Folge des Ohrenunfalls zu bestimmen. Zunächst sind die Veränderungen am Ohre selbst (Trommelfell und Labyrinth) gleich nach der Verletzung ganz andere, als einige Zeit nachher; sodann hat die Erfahrung gezeigt, dass Ohrverletzte kurz nach der Verletzung ein viel günstigeres Hörvermögen zugeben als später, weil sie unter dem Eindruck der Freude, noch so glücklich bei ihrer Beschädigung davon gekommen zu sein, sofort nach dem Unfall richtige Angaben über das Hörvermögen zu machen pflegen, während spätere Angaben über die verbliebene Hörkraft durchweg viel ungünstiger ausfallen.

Der obengenannte Verein hat deshalb in seinen Sitzungen rom 24. November 1901 und 27. April 1902 beschlossen, die Berufsgenossenschaften unter Hinweis anf Obiges zu ersuchen, dafür Bestimmungen zu treffen, dass bei Schädelverletzungen, welche so häufig eine Beeinträchtigung des Hörvermögens zur Folge haben, und bei directen Ohrbeschädigungen ein Ohrenarzt möglichst sofort nach dem Unfall behufs Feststellung des Befundes zugezogen wird.

Es dürfte dies Verfahren sowohl im Interesse des Verletzten als auch im Sinne der Berufsgenossenschaften liegen."

Reinhard schlägt vor, dass die Deutsche otologische Gesellsohaft diese Resolution dadurch unterstützt, dass auch sie an alle Berufsgenossensehaften ein Rundschreiben richtet folgenden Inhaltes:

Die Deutsche otologische Gesellschaft unterstützt die Be- 
schlüsse der westdeutschen Hals- und Ohrenärzte vom 24. November 1901 und 27. April 1902 und empfiehlt den Berufsgenossenschaften bei Schädelverletzungen, welche von Ohrenleiden so häufig begleitet sind, und bei Ohrverletzungen möglichst sofort nach dem Unfall einen Ohrenarzt behufs sofortiger Aufnahme des Befundes hinzuzuziehen."

Die Gesellschaft ist mit diesem Vorschlag einstimmig einverstanden.

III. Killian-Freiburg demonstrirt an drei sehr sorgfältig in Lebensgrösse hergestellten Gypsmodellen seine Radicaloperation chronischer Stirnhöbleneiterungen und führt dabei Folgendes aus:

Seit Jahren habe ich mich bestrebt, die Methoden zur radicalen Beseitigung chronischer Eiterungen in den Stirnhöhlen so weit zu verbessern, dass sie sowohl eine vollständige Heilung herbeiführen, als in cosmetischer Hinsicht befriedigen.

Wie die praktische Erfahrung gelehrt hat, ist bei complicirt gestalteten Stirnhöhlen eine vollständige Ausschaltung des ganzen Hohlraumes nöthig, um zu einem sicheren Ziel zu gelangen. Man erreicht dies durch Resection der vorderen nebst unteren Stirnhöhlenwand und vollständiger Beseitigung der Schleimhaut. Nur dies radicale Vorgehen leistet dafür Gewähr, dass man alle Nebenräume des Sinus frontalis auffindet und sich zugänglich macht. $\mathrm{Da}$ durch vollständige Opferung der vorderen und unteren Stirnhöhle eine wüste Entstellang entstehen würde, so ist es nöthig, den kosmetisch wichtigsten Theil beider zu schonen. Als solchen muss man den oberen Rand der Orbita bezeichnen. Meine Exfahrungen haben mich gelehrt, dass man diesen Rand in Gestalt einer schmalen Knochenspange erhalten kann, ohne dadurch eine Verödung der Stirnhöhle zu verhindern.

Bei jeder rationellen Stirnhöhlenoperation müssen die Siebbeinzellen frei zugänglich gemacht werden, damit diese, soweit sie erkrankt befunden werden - und das ist bei den Stirnzellen Regel - vollständig beseitigt werden können.

Dies führt gleichzeitig zur Anlegung eines für die definitiva Aushebung erforderlichen breiten Weges von der gtirnhüthenwunde zur Nasenhöhle, wenn man darauf bedacht is Hälfte der mittleren Muschel mit zu entfernen.

Den bequemsten Ausgang zum Siebbein ohne f nach liche Entstellung zu riskiren, erhält man durch Re dtion dxes Stirnfortsatzes des Oberkiefers. Der dadurch entehende 
Knochendefect lässt sich bequem mit dem durch Resection der unteren Stirnhöhlenwand entstandenen vereinigen, ohne den $\mathrm{Be}-$ stand der Supraorbitalspange zu gefährden.

Alle genannten Eingriffe lassen sich bequem von einer bogenförmigen Hautwunde aus durchführen, die durch einen innerhalb der Augenbraue und von da in schönem Bogen inmitten der Nasolabialfalte nach abwärts geführten Hautschnitt entsteht. Die primäre Naht dieses Schnittes ermöglicht eine lineare, später kaum sichtbare Narbe. Eine leichte flache Vertiefung der Stirne im Bereiche des operirten Sinus frontalis ist das einzige dauernde Merkmal der stattgefundenen Operation.

Nach diesem Grundplane habe ich im Laufe der letzten Jahre eine Serie von Fällen operirt und bei einem sehr befriedigenden kosmetischen Resultate zur definitiven Heilung gebracht. Es befanden sich Kranke darunter, bei denen die früheren Methoden, selbst wiederholt angewandt, nicht zum Ziele geführt haben.

Die ausführlichen Krankengeschichten und eine eingehende Schilderung der Teehnik meines Verfahrens befindet sich in dem demnächst erscheinenden 1. Heft des XIII. Bandes des Archivs für Laryngologie (1902).

Die demonstrirten Gypsmodelle sind erhältlich bei Instrumentenmacher Fischer-Freiburg i. B.

\section{Discussion:}

Jansen-Berlin: Neu an der Killian'schen Methode ist das Stehenlassen der Orbitalspange, sowie auch der Versuch einer Lappenbildung aus der Nasenschleimhaut. In allen tibrigen Punkten ist die Methode genau dieselbe, wie diejenige, welche ich auch schon seit 3-4 Jahren und länger anwende, und verschiedentlich in Diseussionen über dieses Thema bekannt gegeben habe. Ein Vortheil der Killian'schen Methode ist das Verharren des Margo supraorbitalis in seiner natiurlichen Lage. Infolgedessen scheint das Auge nicht so abgeflacht, ja vorgedrängt, wie nach dem Einsinken der Augenbraue. Selbstverständlich lohnt sich das Erhalten der Orbitalspange nur bei grossen Eiohlen. Dagegen wird bei sehr grossen und tiefen Stirnhöhlen nach Fortnahme der ganzen vorderen Stirnhöhlenwand die Stirne stark zurückgelagert und dadurch das Profil sehr verändert. Das sehen wir anch bei den stereoskopischen Photographien des von Killian doppelseitig operirten Herren. Ich glaube für solche grosse Höhlen erhält man in kosmetischer Beziehung bessere Resultate, 
wenn man einen möglichst grossen Theil der vorderen knöchernen Stirnhöhlenwand als Knochenperiosthautlappen erhält und auf die hintere Stirnhöhlenwand zurüektamponirt. Seit über 2 Jahren habe ich in mehr als 30 Fällen diese Methode angewendet. Noch bessere Resultate wird man vielleicht erzielen, wenn man damit die Erhaltung eines Teiles der Orbitalspange in Zusammenhang mit dem oberen Lide verbindet. Die Erhaltung des Margo supraorbitalis in seiner natürlichen Lage am Schädel giebt mir dagegen zu mancherlei Befürchtungen Anlass. Ich fürchte, die Stirnhöhle kommt nicht vollständig aur Verödung, und das Fortbestehen einer Eiterung oder eine Secretion von Schleim dürfte die Folge sein. Desgleichen fürchte ich, dass besonders bei enormer Ausdehnung der Stirnhöhlen nach Aussen Neigung zur Taschenbildung vorhanden sein wird. Auch ich habe früher geglaubt, dass das orbitale Fettgewebe durch ausgedehnte Verlagerung einen grossen Theil des Raumes der Stirnhöhle einnehmen könne, and habe das in meiner ersten Arbeit im Archiv für Laryngologie auch ausgesprochen. Diese Verlagerung hat aber doch engere Grenzen, als ich früher glaubte. Nur der hintere und fast regelmässig niedrigere Abschnitt der Stirnhöhle am orbitalen Dache dürfte so ausgeführt werden, nicht aber die höheren und mehr medialwärts gelegenen Theile der Stirnhöhle. Das Stehenlassen des Margo supraorbitales in situ wird, wie ich fürchte, den Ueberblick und das Freilegen des ganzen Siebbeines und der Keilbeinhöhle bisweilen recht erschweren. Ich betrachte es aber als einen wesentlichen Theil der Operation, das ganze erkrankte Siebbein zu entfernen. Der von Killian vorgezeigte Wundhaken zur Niederhaltung des durch die Kapsel perforirten orbitalen Fettgewebes scheint mir viel zu schmal. Ich benutze einen breiten schaufelförmigen, aber auch dieser hält das orbitale Fettgewebe vielfach nicht genügend zurück. Mit Hilfe eines Gazetupfers lässt er sich dagegen mit einem schaufelförmigen Wundhaken ganz bequem zurückhalten.

Jens-Hannover hat bisher entweder nur die vordere oder die untere Wand fortgenommen; in beiden Fällen hat es ihm immer Schwierigkeiten gemacht, eine genügend grosse Oeffnung nach der Nase hin anzulegen. Bei der letzten vor wenigen Wochen vorgenommenen Aufmeisselung - Fortnahme der unteren Wand - legte er nun ausserdem mit dem Hohlmeissel eine etwa $3 / 4 \mathrm{~cm}$ breite und $1-1 \frac{1 / 2}{\mathrm{~cm}}$ lange Rinne in der vorderen Wand an (ohne dabei die Weichtheile nach oben hin zu spalten); 
nach Anlegung dieser Rinne war dann das Arbeiten in der Tiefe, speciell in der Gegend des Processus uncinatus, ein viel bequemeres. Es wird sich bei dieser Modification mehr von der unteren Wand erhalten lassen als bisher, und damit ein nachträgliches Einsinken des Bulbus zu vermeiden sein.

Hoffmann-Dresden: Die vollständige Entfernung der vorderen Wand der Stirnhöhle pflegt besonders bei grösseren Höhlen von mehr oder weniger grosser Entstellung gefolgt zu sein. Deshalb ziehe ich derselben die osteoplastische Aufmeisselung der Stirnhöhle vor. Wenn man dadurch keinen genügenden Ueberblick nach unten zu erhält, so kann man zu dem Zweck, den radicalen Schnitt $\mathrm{K} u \mathrm{~h} n \mathrm{t}$ 's nach unten verlängernd, den Rest der vorderen Wand, und wenn nöthig, auch noch einen Theil der unteren subperiostal nach unten umklappen. Breite Drainage nach der Nase, Naht.

Siebenmann-Basel.

\section{Killian-Freiburg: Ein grosses Modell der Nasenhöhle zur Verwendung beim klinischen Unterricht.}

Der klinische Unterricht in der modernen Rhinologie erfordert ein häufiges Eingehen auf den anatomischen Bau der Nasenhöhle. Ganz besonders kommen dabei die Nebenhöhlen in Betracht. Manche diagnostische und therapeutische Maassregeln lassen sich an Tafeln nur schwer verständlich machen. Ich habe daher ein Modell in grossen Dimensionen anfertigen lassen, welches selbst die feineren anatomischen Verhältnisse einem grösseren Auditorium zur Anschauung bringt. Die Nasenmuscheln können herausgenommen werden, sodass ein bequemer Einblick in das System der Nebenhöhlen möglich ist. Dem Modell wurde ein Präparat zu Grunde gelegt, das typische und entwickelungsgeschichtlieh klare Verhältnisse zeigte. Die genauere anatomische Beschreibung ist zu finden in dem Arehiv für Laryngologie, XIII. Band, 1. Heft 1902. Bezugsquelle: Fischer, Univers. Instrumentenmacher, Freiburg i. B.

$\mathrm{V}$. Streit-Königsberg: Operationsmethode zur ausgedehntesten Freilegung der vorderen Pgramidenfläche behufs Aufsuchen tiefliegender Eiterherde. (Aus dem Königsberger Ambulatorium von Herrn Professor Leutert.)

Durch Wegnahme des Jochbogenausläufers, des darüber liegenden Knochens $1 \mathrm{~cm}$ weit von letzterem aus gerechnet, der 
oberen Gehörgangswand, des Ueberganges von oberer und vorderer Gehörgangswand wird die Dura in Form eines Ovals mit $21 / 2 \mathrm{~cm}$ Längs- und $2 \mathrm{~cm}$ Queraxe freigelegt. Hierdurch schaffit man sich einen directen Zugang nach der Pyramidenspitze zu und kann von dieser freigelegten Stelle aus die Dura mit einem elastischen zu diesem Zweeke construirten Hirnspatel bis an die Pyramidenspitze abhebeln. Wenn man sich zu dieser Manipulation reflectirten Lichtes bedient, ist es möglich, jede Phase der Operation zu beobachten. Etwaige sich vorfindende stärkere Verwachsungen zwischen Dura und Knochen sind mit einem als "Raspatoriumeurette" bezeichneten Instrument unter dem Schutz des darüber liegenden Spatels zu durehtrennen.

Diese „Raspatoriumourette" kann man gleichzeitig als. Curette zur Entfernung von cariösem Knochen und Granulationen benutzen. Eine genaue Schilderung der Methode, der Indication für sie u. s. w. wird demnächst in diesem Archiv zu erwarten sein. Schluss der Freitag-Sitzung 6 Uhr.

Am Abend vereinte ein Festmahl die Theilnehmer und ihre Damen mit den Vertretern der Stadt in dem Gartensaal des Casino, bei welchem die herrlichen Gewächse der Mosel und der Saar zur Geltung kamen.

\section{Samstag, den 17. Mai 9 Uhr.}

Geschäftssitzung: Bericht des Schriftführers A. EartmannBerlin.

Für das Tröltsch-Denkmal steht ein sehr grosser Betrag bereit; nach eingelaufenen Nachrichten von Würzburg wird beabsichtigt, dort eine Ohrenklinik zu erbauen. - Die otologische Gesellschaft hält es für am besten, nach Fertigstellung derselben eine Broncebüste unseres Meisters, für welche $5000 \mathrm{Mk}$. ausreichen werden, in der Klinik zur Aufstellung zu bringen. Der Beschluss hierüber wird noch ausgesetzt.

4 Mitglieder sind im vergangenen Jahre gestorben.

$7=\quad=$ ausgetreten.

$7=\quad$ neu eingetreten.

Im Vorjahre war in Breslau bereits beschlossen, der Gesellschaft die Rechte einer juridischen Person za versehaffen; zu diesem Zwecke muss behufs Eintragung in das Vereinsregister eine Satzung eingereioht werden. Ein Entwurf solcher Satzung wird vorgelegt, die Berathung darüber aber bis zum nächsten 
Jahre verschoben. - Es folgt der Kassenbericht von 0. WolffFrankfurt und der Bericht des derzeitigen Bibliothekars Körner-Rostock, welcher um Zuwendungen geeigneter Literatur für die Bibliothek bittet, wie dies bereits in entgegenkommender Weise von Denker-Hagen, Siebenmann-Basel, Joël-Görbersdorf und Hartmann-Berlin geschehen ist. Das Verzeichniss enthält bisher 300 Bände. Es wird zur Anschaffung eines Schrankes eine einmalige Summe von $300 \mathrm{Mk}$. bewilligt.

Bei der Wahl des Ausschusses hat Bürkner eine Wiederwahl aus Gesundheitsrïcksichten abgelehnt; die übrigen Mitglieder des Ausschusses Lucae, Bezold, Habermann, Hartmann, Körner, Kretsehmann, Siebenmann, Wolff werden wieder, Passow-Heidelberg neu gewählt.

Als Ort der nächsten Versammlung werden Berlin und Wiesbaden vorgeschlagen, Wiesbaden wird gewählt. Rudloff begrüsst die Theilnehmer für die alte, bewährte Congressstadt.

VI. Ostmann-Marburg: Zur Analyse der Hörstörung bea der acuten perforirten Mittelohrentzündung.

Der Vortragende berichtet äber die Ergebnisse seiner Stimmgabeluntersuchungen, die er im Laufe der letzten 3 Jahre bei 9 Fällen von acuter perforirter Mittelobrentzündung vorgenommen hat.

Die Analyse bestand darin, dass mit der continuirlichen Tonreihe während des Ablaufes der Exkrankungen täglich oder in Intervallen von einigen Tagen:

1. der Hörumfang,

2. das Hörrelief innerhalb der gefundenen Hörstreeke,

3. der Ausfall des Weber'sehen und

4. der Ausfall des Rinne'schen Versuches bestimmt wird.

Der Vortragende erläutert seine Ausführungen an Tafeln, auf denen die Resultate der einzelnen Versuche graphiseh dargestellt sind.

Der Vortrag wird demnäehst in einer der Fachzeitschriften in erweiterter Form veröffentlicht werden.

Grosser Beifall lohnte die überaus fleissige und alle Hörer auf's höchste befriedigende Arbeit des Vortragenden.

Discussion:

Bezold bestätigt die Beobachtungen Ostmann's.

Scheibe-München: Schlechtes Gehör für Galtonpfeife er- 
Bericht über die Versammlung der deutschen otologischen Gesellschaft. 107

giebt böse Prognose und indicirt frühzeitige Eröffnung des Processus mast.

Hoffmann-Dresden fragt wegen subjectiver Geräusche. VII. Kümmmel-Bresla u empfiehlt an der Hand eines aufgestellten Apparates das Telephon als einfachen Hörmesses für hohe und tiefe Töne.

VIII. Eschweiler-Bonn: Veber Nystagmus bei einseitiger Labyrinthlosigkeit.

Eschweiler demonstrirt einen Labyrinthsequester, an dem die Cochlea, das Vestibulum und Theile der Bogengänge deutlich zu sehen sind. Die Untersuchung des 22jährigen Kranken ergab Nystagmus beim Blick nach der gesunden Seite, wenn der Kranke von der gesunden zur kranken Seite um seine Verticalaxe gedreht worden war. Darin besteht eine Abweichung von drei Fällen Wanner's. Eschweiler ist der Ansicht, dass durch Beobachtung von Nystagmus bei einseitig Labyrinthlosen dann erst eine Erklärung für die Wirkung der Bogengänge auf die Augenmuskeln gegeben werden kann, wenn festgestellt ist, ob und wie der Nystagmus dureh Ablenkung der Bulbi in gewisser Richtung zu Stande kommt.

Discusion:

Bezold hält die Zahl der Fälle für zu klein, um eine definitive Entscheidung zu fällen.

IX. Eschweiler-Bonn: Demonstrationen zur Entwicklung der Mittelohrmusculatur.

Das Material waren Schweineembryonen von 10,5 bis $53 \mathrm{~mm}$ Scheitel-Steisslänge. Eschweiler ist zu der Ueberzengung gekommen, dass im primitiven Zustand eine untrennbare Blastemmasse die Kaumusculatur, den Merkel'schen Knorpel, die Gehörknöchelehen und den Musculus tensor tympani enthält. Zunächst wächst diese relativ kurze Blastemmasse oral-aboral, resp. ventrodorsalwärts aus, wobei die Differenzirung oral beginnt und aboralwärts fortschreitet. Die Ueberleitung der Kaumusculatur in den Musculus tensor tympani vermittelt der Museulus tensor. vel. palatini.

X. Rudloff-Wiesbaden: Demonstration mikroskopischer Präparate von Veränderungen im Tubenknorpel bei Hyperplasie der Rachenmandel.

Die Präparate, welehe jüngeren Individuen (14 und 17 Jahre alt) entstammen, zeigen 
1. Veränderungen der Grundsubstanz.

2. Veränderungen der Knorpelzellen.

$\mathrm{Zu}$ 1. Beim Neugeborenen ist der Tubenknorpel hyalin, beim Erwachsenen dagegen hat die Grundsubstanz fibrillären Bau, in welchem Lebensalter aber die Umwandlung erfolgt, ist aus den Angaben der Literatur nicht ersichtlich, nur Urbantsehitseh hebt hervor, dass die streifige Grundsubstanz erst nach iberschrittenem Kindesalter häufig wird. Es können also auch bei jüngeren Individuen in der Grundsubstanz Fasern auftreten, es wäre aber auch denkbar, dass die Fasern in den untersuchten Knorpelstücken deshalb so frühzeitig aufgetreten sind, weil die Schleimbaut von adenoidem Gewebe reichlich durchsetzt ist. An diese Möglichkeit lässt das massenhafte Vorkommen von Fasern in dem jugendlichen Knorpel denken, ferner die vorhandenen Formveränderungen der Zellen.

$\mathrm{Zu}$ 2. Die Zellen sind spindelförmig; sternförmig oder Knochenzellen ähnlich; dabei kann die Knochenzellenböhle erhalten oder geschwunden sein.

Formveränderungen der Zellen in der Weise, wie die Präparate es zeigen, sind als degenerative Veränderungen im Tubenknorpel bisher noch nicht beschrieben. Da diese Formveränderung der Zellen bei beiden Präparaten in gleicher Weise vorhanden ist, und da bei beiden Knorpelstiucken die umgebende Schleimhaut erkrankt ist, so liegt es nahe, diese Formveränderung der Zellen mit der Erkrankung der Schleimhaut in ursächlichen Zusammenhang zu bringen.

XI. Siebenmann-Basel: Ueber Hypoplasie und Faltenbildung der häutigen Labyrinthwand der Pars inferior als Ursache von angeborener Taubstummheit (mit Demonstration von Tafeln und mikroskopischen Präparaten).

Das knöcherne Labyrinth ist normal und der Acusticusstamm nicht oder nur unmerklich verdünnt, das Ganglion cochleare ist hochgradig atrophisch (Hypoplasie), das Corti'sehe Organ degenerirt und die Nervenfasern zwischen Ganglion und Papilla acustica sind nur spurweise vorhanden.

Von Stelle zu Stelle ist der Ductus cochlearis auffällig erweitert : einerseits inserirt sich die Reiss ne r'sehe Membran streckenweise abnorm hoch oben, andererseits hat sich die Epitheldecke der Stria vascularis blasen- oder faltenförmig von iher Unterlage abgehoben und zwar in allen Windungen auf grössere und klei- 
nere Strecken. An vereinzelten Stellen reichen diese Falten bis zur Lamina spiralis herab, wo sie mit den epithelialen Gebilden verkleben und sie auf mannigfache Weise deformiren und dislociren. - Auch die Sacculuswände sind in Falten gelegt und derart auf einander gesunken, dass das Macula-epithel durchweg degenerirt und das freie Lumen des Sacculus fast gänzlich aufgehoben ist. - Rechts und links sind die Verhältnisse ganz die nämlichen, nur wechselt der Sitz und die Ausbildung der Faltenbildung etwas. - Zwei gleiche Befunde hat Scheibe beschrieben; doch sind in dem einen dieser Fälle die Vorhofgebilde normal. Aehnliche Veränderungen fand G. Alexander und Rawitz bei einer tauben albinotischen Katze; doch betraf wenigstens im ersteren dieser Befunde die Faltenbildung in der Schnecke nicht die Stria sondern die Reissner'sche Membran. Mit Sicherheit kann schon jetzt behauptet werden, dass die excessive Faltenbildung und der Collaps der embryonalen Labyrinthwand in der Pathogenese der angeborenen (hereditären) Taubstummheit eine hervorragende Rolle spielen.

Der Fall wird später ausführlich und mit Abbildungen durch Dr. Oppik ofer in der Zeitschrift für Obrenheilkunde publicirt.

\section{Bloch-Freiburg i. Br.: Was nennen wir einen hohen}

\section{Gaumen?}

Vortragender wendet sich gegen die unter der Leitung Siebenmann's ausgeführte Arbeit von E. Fränkel: Der abnorme Hochstand des Gaumens, Jahr 1896, deren Ergebniss, von seinem Lehrer acceptirt, dahin lantet, dass bei habitueller Mundathmung: in Folge von Hyperplasie der Rachenmandel im Durchschnitt der Gaumen nicht höher sei als bei normaler Nasenathmung.

Fränkel's Material ist nicht gross genug, um die Frage zu entscheiden, und seine Messungen des Gaumen-Höhenbreitenindex sind nicht an der geeignetsten Stelle ausgeführt. Fränkel hat am I. Prämolaren den Index bestimmt, während er in der Mehrzahl der Fälle hintem dem II. Prämolaren seinen grössten Werth erreicht. Ferner hat er Kinder und Erwachsene gleichmässig statistisch verwerthet, obwohl bei Kindern in der I. Dentition, wie bekannt, die bezüglichen Veränderungen noch nicht so weit vorgeschritten sind als später. - Ebenso müssen diejenigen seiner Fälle ausgeschieden werden, in welchen die Mundathmung erst nach beendigtem Zahnwechsel, im 20., im 18., oder im 16. Jahre eintrat. 
Die Frage, welcher Gaumenindex dem hohen Gaumen entspreche, beantwortet F r änkel dadurch, dass er 43 Personen ohne Mundathmung und ohne vergrösserte Rachenmandeln misst und die Summe dieser 43 Gaumenindices dureh 43 theilt. Die resultirende Ziffer gilt ihm als Normalindex, was darüber liegt, zählt zum hohen Gaumen.

Dieser Methode gegenüber geht Vortragender in der Weise vor, dass er in seinen 600 bis jetzt gemessenen Fällen zunächst die Gaumenhöhe sehätzt und darauf den Index dureh Messung bestimmt. Dabei findet sich, dass der als hoch oder wenigstens als höher geschätzte Gaumen einen Index von $>\mathbf{5 8}$ besitzt. Und während Erwachsene - um von Kindern mit Vilehzähnen und solchen im Jahreswechsel hier abzusehen - mit Nasenathmung im Mittel unter 286 Fällen einen Gaumenindex von 52,5 aufweisen, haben die 199 gemessenen erwachsenen Mundathmer einen durchschnittlichen Gaumenindex von 63,8 .

Damit ist die Siebenmann-Fränkel'sche Behauptung: widerlegt.

\section{Jansen-Berlin: Neue Erfahrungen über chronische Nebenhöhleneiterungen der Nase.}

Bei ehronischen Eiterungen der Stirnhöhlen habe ich meine Operationsmethode etwas modificirt und seit etwa 2 Jahren in über 30 Fällen in etwa folgender Weise verfahren. Nach Eröffnen der Stirnhöhle unter dem Supraorbitalrande fraise ich die vordere Stirnhöhlenwand von der Stirnhöhle aus am medialen und am lateralen Rande von der Umgebung ab, so hoch hinauf als möglich ist. Mit besonderer Sorgfalt muss darauf geachtet werden, dass die Weichtheile sich von der vorderen Wand nicht loslösen. Darauf fasse ich mit einer breiten Knochenzange die vordere Stirnhöhlenwand und breche sie möglichst oben ab. Dieser Knochenperiosthautlappen wird mit einem Stückchen Gaze geschützt und mit einem breiten Wundhaken abgehoben. Jetzt ist die Stirnhöhle gut übersichtlich, und die überhängenden Knochenränder werden sorgfältig abgemeisselt. Dieser Knochenperiosthautlappen legt sich bald auf die hintere Stirnhöhlenwand und vermindert das Zurückweichen der Stirn, bisweilen sogar in einem merkwürdig hohen Grade. Der kosmetische Effect ist weit besser als nach der vollständigem Fortnahme der vorderen Wand. Ich erlaube mir, Ihnen verschiedene stereoskopische Aufnahmen zu zeigen, die nach allen möglichen Richtungen ausgeführt 
sind, ganz besonders auch in derjenigen Position, in der die Entstellung am meisten in die Erscheinung tritt. Die Operation wird durch diese Methode etwas verlängert und dauert zwischen dreiviertel bis anderthalb Stunden, wobei ich dann die Stirnhöhle, das Siebbein und die Keilbeinhöhle, eventuell anch anf beiden Seiten in breitester Weise eröffnet habe. Die Ausheilung ist in günstigsten Fällen schon in 6 Wochen erfolgt, hat aber ofters auch bis zu einem halben Jahre gedauert. Seit etwa einem halben Jahre habe ich auch bei der Operation der Kieferhöhlenempyeme eine Modifieation vorgenommen. Wenn ich bei der Eröffnung der Kieferhöhle solche Verhältnisse vorfand, dass ich annehmen durfte, es könne eine Rückbildung der erkrankten Sehleimhaut zur normalen eintreten, so habe ich mich bestrebt, die Schleimhant so weit als möglich in der Höhle zu belassen und nach Herstellung einer sehr breiten und dauernden Communication nach der Nase die Eingangsöffnung in der Fossa eanina primär durch Naht zu verschliessen. Wenn das Siebbein krank war, so habe ich ebenso, wie ich es frither besehrieben habe, von der Kieferhöhle aus auch die Siebbeinzellen, so weit ich es vermochte, eröffnet und entfernt und, wenn nöthig, die Eröffnung der Kẹilbeinhöhle durch Fortnahme der vorderen eventuell auch der unteren Wand angeschlossen. Wenn ich die primäre Vereinigung der Eingangsöfnung in der Fossa canina ausfuhren will, so verzichte ich meist auf die vollständige Entfernung der vorderen Kieferhöhlenwand, insbesondere habe ich auf die exacte Freilegung der Jochbeinbucht und des Bodens der Kieferhöhle verzichtet. Im Uebrigen habe ich aber doch fast die ganze vordere Wand fortgenommen. An die Eröffnung der Kieferhöhle schliesse ich die Fortnahme der knöchernen Nasenwand, während ich aus der Schleimhaut der Nasenwand einen möglichst grossen Lappen bilde, in der Regel mit der Basis nach vorne, bisweilen auch mit der Basis nach unten, und nach Abpräpariren von der Muschel sohlage ich diesen Lappen in die Höhle hinein und tamponire ihn, wenn er die Basis unten hat, auf den Boden oder armire ihn mit 1 oder 2 Seidenfäden und hefte diesen Lappen nach dem Zunähen der Eingangsöffnung an die vordere Kieferhöhlenwand, d. h. an die Wangensehleimhant fest. Die untere Muschel lasse ich in der Regel stehen; wenn sie gross ist, exeidire ich den unteren Rand. Wenn ich das Siebbein herausnehme, entferne ich in der Regel auch die untere Muschel. Jetzt habe jeh eine breite Communication nach der Nase, welehe sich 
in Folge der Lappenbildung nicht mehr verschliessen, ja nicht mehr verkleinern kann, während ich ohne diese Lappenbildung selbst die allergrössten Defecte zu ganz minimalen Fisteln sich verengern sah. Will man eine grössere Uebersicht von der Nase aus über die Kieferhöhle haben, so muss man auch die untere Muschel amputiren; darauf habe ich aber in der Regel verzichtet. Der Kranke ist bald in der Lage, mit einem entsprechend gebogenen Röhrchen die Ausspülungen von der Nase aus selbst vorzunehmen. Im Anfang ist die Eiterung sehr reichlich, nach und nach wird sie geringer entsprechend der Abnahme der Schwellung der Schleimhaut. Ich habe die Methode noch nicht lange genug ausgeführt, um ein Urtheil über den dauernden Werth derselben zu haben. Nicht ganz leicht ist zuweilen das Herausziehen der Jodoformgazestreifen aus der Kieferhöhle zur Nase heraus beim ersten Verbandwechsel. Bisher bin ich mit dem Resultat dieser Operationsmethode so zufrieden, dass ich dieselbe weiter verfolgen werde, und ich glaube, ich werde zu Gunsten dieser Operationsmethode sowohl die Anbohrung von der Alveole aus mehr einschränken wie auch die persistenten Oeffnungen in der Fossa canina.

\section{Brieger-Breslau: Ueber das Vorkommen otogener Meningitis serosa.}

Das Vorkommen einer rein serösen otogenen Meningitis ist anatomisch nicht bewiesen. Die zur Beweisführung herangezogenen klinischen Beobachtungen dürfen als beweiskräftig nur dann gelten, wenn in dem Krankheitsbild die Erscheinungen der intracraniellen Drucksteigerung prävaliren, und wenn diese Erscheinungen auf eine intracraniellen Druck erniedrigende Therapie so unmittelbar rüokgängig werden, dass der Causalzusammenhang zwischen dieser Druckentlastung und dem Rüickgang der Symptome unzweifelhaft ist. Fälle dieser Art, bei denen u. A. basale, in einem Falle auch eine nucleäre Augenmuskellähmung auf Lumbalpunction wieder rückgängig wurde, werden erörtert. Als beweisend sind aber auch solche anscheinend einwandsfreie Beobachtungen nur dann anzuerkennen, wenn bei dem zur Druckentlastung vorgenommenen Eingriff das Vorhandensein der Drucksteigerung exact nachgewiesen ist.

Die Liquorvermehrung, die dem Krankheitsbilde der Meningitis serosa zu Grunde liegt, scheint hanptsächlich durch Labyrintheiterungen indicirt zu sein. Die otogene Meningitis serosa 
ist kein idiopathischer Process, sondern in der Hauptsache ein Symptom labyrinthärer Eiterung. Die Vermehrung der Liquorproduction scheint durch Einwirkung aus der Perilymphe in den Liquor gelangenden toxischen Stoffe bedingt zu sein. Dafür sprechen Thierversuche, welche sogar die Möglichkeit des Vorkommens toxisch bedingter eitriger Meningitiden zu ergeben scheinen.

Das Vorkommen einer ansschliesslichen oder so überwiegenden Vermehrung des Hirnwassers innerhalb der Hirnhöhlen, dass es zu einem Abschluss gegen den Arachnoidealraum und somit zu ausschliesslichem Hydrocephalus internus käme, ist nicht bewiesen und an sich unwahrseheinlich. In den Fällen, in denen sich an labyrinthäre Eiterung eine Vermehrung des Liquors anschliesst, betrifft diese Vermehrung das ganze Liquor führende System gleichmässig.

Damit vereinfacht sich die Therapie dieser Zustände. Wo nicht aus diagnostischen Rucksichten probatorisch eine directe Eröffnung des Arachnoidealraums in der Nachbarschaft des primären Eiterherdes vorgenommen wird, ist die Lumbalpunction zum Zwecke der Druckentlastung in erster Reihe vorzunehmen. Die wichtigste Aufgabe der Therapie bleibt auch hier wieder die Aussehaltung des primären Herdes, welcher zur Entwickelung der Erscheinungen der Meningitis serosa geführt hat.

XV. Kretsehmann-Magdeburg demonstrirt einen rechtwinkligen Handgriff für die Welle am Elektromotor bei Operationen in der Nase, Kieferhöhle u. s. w.

\section{Rudloff-Wiesbaden demonstrirt}

1. ein sterilisirbares Ohrbesteck zum Gebrauch bei Besuchen von Ohrerkrankten, besonders nach Infectionskrankheiten. Das Besteck hat die Grösse eines Cigarrenetuis aus vernickeltem Metall, enthält ausser Spiegel, Kniepincette, Ohrtrichter, Paracentesennadel, Furunkelmesser und Sonde, welche Instrumente leicht herausgenommen und, ebenso wie das Etui, leicht desinficirt werden können.

2. Einen bequemen stumpfen $W$ undwinkelhaken zum freien Klarlegen des Fossa canina.

Diese Instrumente werden von der Firma W. Holzhauer in Marburg a. d. Lahn gefertigt.

Nach dem Schluss der Sitzung $3^{1 / 2}$ Uhr fand eine BesichtiArchiv f. Ohrenheilkunde. LVI. Bd. 
114 VI. REINHARD, Versammlung der deutschen otolog. Gesellschaft.

gung der sehenswerthen und gut erhaltenen Alterthümer Triers statt, von denen das vor Kurzem errichtete Museum mit seinen hervorragenden Ausgrabungen, welche an das Nationalmuseum in Neapel erinnern, die Kaiserburg und die römischen Bäder an erster Stelle zu nennen sind. Der Abend vereinigte alle Theilnehmer auf dem am linken Moselufer am Bergabhang gelegenen Weisshaus, von wo man einen herrlichen Ueberblick über die gastfreie alte Moselstadt genoss. 